\title{
BMJ Open Gout and the risk of advanced chronic kidney disease in the UK health system: a national cohort study
}

\author{
Austin G Stack, ${ }^{1}$ Michelle Elizabeth Johnson, ${ }^{2}$ Betina Blak, ${ }^{3}$ Alyssa Klein, ${ }^{4}$ \\ Lewis Carpenter, ${ }^{2}$ Robert Morlock, ${ }^{5}$ Andrew R Maguire, ${ }^{2}$ Victoria L Parsons ${ }^{2}$
}

To cite: Stack AG, Johnson ME, Blak B, et al. Gout and the risk of advanced chronic kidney disease in the UK health system: a national cohort study. BMJ Open 2019;9:e031550. doi:10.1136/ bmjopen-2019-031550

- Prepublication history and additional material for this paper are available online. To view these files, please visit the journal online (http://dx.doi org/10.1136/bmjopen-2019031550).

The results of this study were presented at the Annual Scientific Meetings of the American College of Rheumatology in 2016 and the European Renal Association \& European Dialysis and Transplantation (ERA-EDTA) Meeting in 2017.

Received 08 May 2019 Revised 11 July 2019 Accepted 29 July 2019

Check for updates

(c) Author(s) (or their employer(s)) 2019. Re-use permitted under CC BY-NC. No commercial re-use. See rights and permissions. Published by BMJ.

For numbered affiliations see end of article.

Correspondence to

Dr Austin G Stack;

austin.stack@ul.ie

\section{ABSTRACT}

Objective Evaluate the association between gout and risk of advanced chronic kidney disease (CKD).

Design Retrospective matched cohort study.

Setting UK Clinical Practice Research Datalink.

Participants The analysis included data for 68897 patients with gout and 554964 matched patients without gout. Patients were aged $\geq 18$ years, registered at UK practices, had $\geq 12$ months of clinical data and had data linked with Hospital Episode Statistics. Patients were excluded for history of advanced CKD, juvenile gout, cancer, HIV, tumour lysis syndrome, Lesch-Nyhan syndrome or familial Mediterranean fever.

Primary and secondary outcome measures Advanced CKD was defined as first occurrence of: (1) dialysis, kidney transplant, diagnosis of end-stage kidney disease (ESKD) or stage 5 CKD (diagnostic codes in Read system or International Classification of Diseases, Tenth Revision); (2) estimated glomerular filtration rate (eGFR) $<10 \mathrm{~mL} /$ $\mathrm{min} / 1.73 \mathrm{~m}^{2}$; (3) doubling of serum creatinine from baseline and (4) death associated with CKD.

Results Advanced CKD incidence was higher for patients with gout (8.54 per 1000 patient-years; $95 \% \mathrm{Cl} 8.26$ to $8.83)$ versus without gout $(4.08 ; 95 \% \mathrm{Cl} 4.00$ to 4.16$)$. Gout was associated with higher advanced CKD risk in both unadjusted analysis (HR, 2.00; $95 \% \mathrm{Cl} 1.92$ to 2.07) and after adjustment ( $\mathrm{HR}, 1.29 ; 95 \% \mathrm{Cl} 1.23$ to 1.35). Association was strongest for ESKD (HR, 2.13; $95 \% \mathrm{Cl} 1.73$ to 2.61 ) and was present for eGFR $<10 \mathrm{~mL} /$ $\mathrm{min} / 1.73 \mathrm{~m}^{2}(\mathrm{HR}, 1.45 ; 95 \% \mathrm{Cl} 1.30$ to 1.61$)$ and serum creatinine doubling ( $\mathrm{HR}, 1.13 ; 95 \% \mathrm{Cl} 1.08$ to 1.19 ) but not CKD-associated death (HR, 1.14; $95 \% \mathrm{Cl} 0.99$ to 1.31). Association of gout with advanced CKD was replicated in propensity-score matched analysis ( $\mathrm{HR}, 1.23 ; 95 \% \mathrm{Cl} 1.17$ to 1.29) and analysis limited to patients with incident gout (HR, 1.28; 95\% Cl 1.22 to 1.35).

Conclusions Gout is associated with elevated risk of CKD progression. Future studies should investigate whether controlling gout is protective and reduces CKD risk.

\section{INTRODUCTION}

Chronic kidney disease (CKD) is a major public health epidemic, with at least 1 in 10 individuals having some degree of kidney impairment, contributing substantially to adverse clinical and economic outcomes. ${ }^{1-3}$ While attention has traditionally focused on

\section{Strengths and limitations of this study}

The large sample size of more than 600000 patients and moderate follow-up period provided a powerful mechanism to examine multiple outcomes.

- The study cohort analysed data from Clinical Practice Research Datalink, ensuring that results are broadly generalisable to primary care practices in the UK health system population.

- Comprehensive data on several confounders were accounted for in the analysis, but these did not explain the strong relationship between gout and risk of advanced chronic kidney disease.

the identification and treatment of common CKD risk factors such as hypertension, diabetes and smoking, there is increasing interest by the scientific community in the role of novel risk factors, such as uric acid and gout, which may independently contribute to disease progression and higher rates of end-stage kidney disease (ESKD). ${ }^{4-6}$ Evidence has emerged that the burden of gout and hyperuricaemia is high in the general population and increases with progressive CKD. ${ }^{7}$ Importantly, a large proportion of these patients remain undertreated and continue to have uncontrolled gout. ${ }^{8}$

Gout is a common inflammatory arthritis caused by deposition of monosodium urate crystals in joints and as a consequence of longstanding asymptomatic hyperuricaemia. Despite advances in clinical care, gout remains poorly managed in the general population with only one-third of patients receiving treatment with urate-lowering therapy (ULT) ${ }^{9}$ and less than $50 \%$ of patients achieving gout control. ${ }^{10-13}$ Experimental studies have convincingly shown that elevated uric acid levels initiate renal injury through intraglomerular hypertension and the development of tubulointerstitial fibrosis. ${ }^{14}{ }^{15}$ Prospective observational studies have yielded strong independent associations 
between hyperuricaemia and kidney disease progression with elevated risks for CKD and kidney failure. ${ }^{16-18}$ It is plausible that gout may further increase the risk of CKD compared with hyperuricaemia alone, potentially mediated by higher urate burden, longer periods of exposure, repeated cycles of systemic inflammation from gout flares and greater exposure to non-steroidal anti-inflammatory drugs (NSAIDs) ${ }^{19-21} \mathrm{~A}$ better understanding of gout and its potential for increasing the risk of CKD is warranted.

The primary objective of this study was to evaluate the association of gout with the risk of advanced CKD in the UK health system. Advanced CKD was defined as the need for long-term dialysis or kidney transplant, progression to a sustained estimated glomerular filtration rate (eGFR) $<10 \mathrm{~mL} / \mathrm{min} / 1.73 \mathrm{~m}^{2}$, doubling of serum creatinine from baseline and death associated with CKD.

\section{METHODS}

\section{Setting and study design}

This matched cohort study was based in the UK health system, using primary care data from the UK Clinical Practice Research Datalink (CPRD), linked with hospital admission data from Hospital Episode Statistics (HES) and death certificate data from Office for National Statistics (ONS). This study is based in part on data from the CPRD GOLD database, obtained under license from the UK Medicines and Healthcare products Regulatory Agency (MHRA). The data are provided by patients and collected by the National Health Service as part of their care and support (HES and ONS data, Copyright 2018, reused with the permission of The Health and Social Care Information Centre. All rights reserved). The interpretation and conclusions contained in this report are those of the authors alone.

\section{Data sources}

The CPRD contains anonymised information recorded during routine clinical practice including clinical



Figure 1 Study participant flow diagram. CKD, chronic kidney disease; CPRD, Clinical Practice Research Datalink; eGFR, estimated glomerular filtration rate; HES, Hospital Episode Statistics; ONS, Office for National Statistics; ULT, urate-lowering therapy. diagnoses, prescriptions and laboratory data from over 11 million patients attending 674 general practices across the UK. ${ }^{22}$ HES captured hospitalisation information on all hospitalisation episodes in England. Linked HES data are available for approximately $70 \%$ of CPRD practices in England. ${ }^{22}$ CPRD is also linked to death information from the ONS and provides detailed information on date and cause of death. The Read code system is used for coding diagnoses in CPRD while the International Classification of Diseases, Tenth Revision (ICD-10) system is used in HES and ONS data. Data were available until July 2015 for CPRD, April 2014 for HES and May 2014 for ONS.

\section{Study population}

The study population included adult patients in CPRD from 1 January 2000 to 31 March 2013 who were classified by the presence (exposed cohort) or absence (unexposed cohort) of gout. A diagnosis of gout was based on a clinical record of gout or prescription of ULT in CPRD. The index date in the exposed group was defined as the date of gout diagnosis between 1 January 2000 and 31 March 2013, based on the date of first gout disease record or the date of first treatment with a ULT during this period. An initial matching strategy was used to ensure patients were assessed at a similar point in time (from index date) and to balance demographic characteristics between gout and non-gout patients (ratio 1:up to 10), with matching on year of birth, sex, registered general practice and availability of data linkage with HES and ONS data. Eligible non-gout patients were randomly assigned (without replacement) to the gout patients and allocated the index date of their matched gout patient. Non-gout patients who developed incident gout during follow-up were then also assigned to the gout cohort (with a new index date from the gout diagnosis), that is, there were time-varying exposures.

Patients were included in the study if they were: aged $\geq 18$ years at index date, had at least 12 months of clinical data prior to the index date, were registered at practices in England and had data linked with HES. Patients were excluded if they had history of advanced CKD (defined as treatment with dialysis, recipient of kidney transplant, stage 5 CKD or eGFR $<15 \mathrm{~mL} / \mathrm{min} / 1.73 \mathrm{~m}^{2}$ on or before index date). ${ }^{23-25}$ Additionally, patients were excluded if there was a history of juvenile gout, cancer, HIV, tumour lysis syndrome, Lesch-Nyhan syndrome or familial Mediterranean fever. The flow diagram for cohort construction is shown in figure 1. There were 205295 patients with gout between 1 January 2000 and 31 March 2013 in CPRD. Of these, 112009 met the eligibility criteria and were matched to 1035552 patients without gout, of whom 900462 met eligibility criteria (figure 1). For the primary analysis, 68897 patients with gout were matched with 554964 patients without gout. For sensitivity analyses, two additional cohorts were constructed to validate the results. First, we created a subgroup from the original gout cohort and propensity score-matched patients with gout $(\mathrm{n}=64420)$ with those without $(\mathrm{n}=286110)$. Second, we constructed an incident gout cohort in which we 


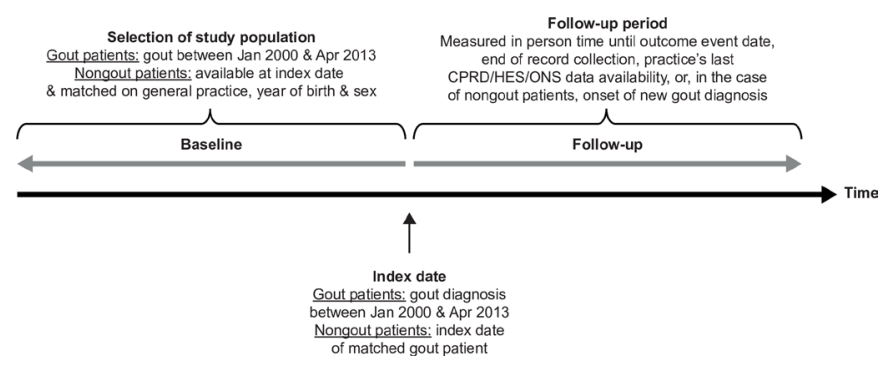

Figure 2 Illustration of study population selection and study periods. CPRD, Clinical Practice Research Datalink; HES, Hospital Episode Statistics; ONS, Office for National Statistics.

compared 53006 patients with a new diagnosis of gout to 432720 patients without gout.

Follow-up data were available until the end of patient's record collection, practice's last CPRD/HES/ONS data availability and outcome event date or, in the case of the non-gout cohort, the date of onset of new gout diagnosis. The study population and study periods are illustrated in figure 2.

\section{Primary outcome}

Advanced CKD, a composite outcome, was defined as first occurrence of: (1) ESKD, defined as long-term dialysis, kidney transplant, diagnosis of ESKD or stage 5 CKD (from diagnostic codes in Read system or ICD-10); (2) eGFR $<10 \mathrm{~mL} / \mathrm{min} / 1.73 \mathrm{~m}^{2}$ (from laboratory results in CPRD); (3) doubling of serum creatinine from baseline from laboratory data in CPRD and (4) death associated with CKD (from ONS mortality data). Deaths were determined to be associated with CKD if one or more ICD-10 code identifying CKD was recorded in any of the causeof-death fields (see list of ICD-10 codes in online supplementary material). An eGFR $<10 \mathrm{~mL} / \mathrm{min} / 1.73 \mathrm{~m}^{2}$ was chosen as an outcome instead of $<15 \mathrm{~mL} / \mathrm{min} / 1.73 \mathrm{~m}^{2}$ (which is a component of kidney failure according to Kidney Disease Improving Global Outcomes clinical guidelines ${ }^{26}$ ) to more precisely capture kidney failure. In the UK, most patients initiate dialysis at an eGFR $<10 \mathrm{~mL}$ / $\min / 1.73 \mathrm{~m}^{2}{ }^{27}$ The inclusion of creatinine doubling captures the progression of $\mathrm{CKD}$, which would have been excluded had outcomes been restricted to those of ESKD.

\section{Confounders and other measured covariates}

The following variables were included in our analyses: demographic factors (age, sex), socioeconomic status (Townsend score of social deprivation), comorbid conditions (diabetes, hypertension, myocardial infarction, angina, heart failure, ischaemic heart disease, coronary artery disease, peripheral arterial disease, stroke, transient ischaemic attack, stage of CKD, acute kidney injury, peptic ulcer disease, rheumatic disease, obesity, depression, dementia) and lifestyle traits (smoking status, alcohol use, history of drug addiction). An age-adjusted Charlson Comorbidity Index was used to measure disease severity. ${ }^{28}$ Data on nephrotoxic medications, NSAIDs and drugs that alter serum creatinine levels (ACE inhibitors, angiotensin-receptor blockers (ARB), diuretics, corticosteroids, aspirin) were also considered as potential confounders in exposure-outcomes relationship. Serum creatinine values were used to estimate kidney function through calculation of eGFR using the Chronic Kidney Disease Epidemiology Collaborative equation. ${ }^{23-25}$ Data on all patient characteristics were captured during any time prior to index date and were considered for inclusion in the multivariable models for adjustment.

\section{Statistical methods}

Incidence rates expressed as events divided by patientyears were calculated for advanced CKD and its four components and reported per 1000 patient-years. Univariable and multivariable marginal Cox proportional hazards models were developed to estimate unadjusted and adjusted HRs and 95\% CIs of advanced CKD associated with gout. Models accounted for the matched cohorts (therefore differences in age, sex, registered clinical practice, number of matched controls) and intracluster dependence introduced by patients who developed incident gout over time and subsequently reclassified from the non-gout to the gout cohort (ie, time-varying exposures). The models of each component of the primary outcome also accounted for the competing risk of the other components. Adjusted models accounted for baseline differences in patient characteristics including prevalent comorbid conditions, social deprivation status, lifestyle traits, clinical measurements and prescribed medications (ACE, ARB, NSAIDs, corticosteroids, thiazide diuretics and aspirin). A manual backwards selection approach with a $5 \%$ significance level was used in model development. Model assumptions were assessed using standard residual-based procedures, and potential collinearity between covariates was investigated (all covariates had a variance inflation factor $<4$ and were therefore retained in the model). Subgroups of clinically important characteristics were explored to determine whether they altered the association between gout and advanced CKD. The following characteristics were selected a priori as clinically important: diabetes, heart failure and CKD stage. Stratum-specific HRs of advanced CKD were computed for each subgroup for patients with and without gout. No imputation was performed on missing clinical values; the extent of missing values was presented in descriptive analyses and then excluded from the statistical models. All statistical analyses were conducted using SAS V.9.4.

\section{Sensitivity analyses}

Several sensitivity analyses were conducted to evaluate the robustness of our findings. First, to exclude potential bias associated with a diagnosis of prevalent gout (included in gout cohort with a history of gout), we restricted the study cohort to patients who developed incident gout (ie, index date was their first ever record of gout) and repeated the entire analysis. Second, the association between gout and advanced CKD was explored in a propensity-score matched analysis to further balance patient characteristics 
between groups and reduce selection bias. Patients with gout were matched with up to five patients without gout based on their probability of being assigned to the gout cohort using propensity scores derived from a logistic regression model using a nearest neighbour matching with a calliper of 0.2 .

\section{Patient and public involvement}

We did not involve patients or the public in our work.

\section{RESULTS}

\section{Patient characteristics}

The study cohort included 623861 patients with and without gout followed for a median of 3.68 years (see online supplementary table 1 ). The prevalence of known risk factors for kidney disease, including hypertension, diabetes, hyperlipidaemia, ischaemic heart disease, benign prostatic hyperplasia, pre-existing CKD and history of acute kidney injury, was more common in patients with gout than without. Moreover, these patients were more often prescribed reno-protective medications, diuretics and NSAIDs. While patients were matched on year of birth and sex, the gout cohort was marginally older overall than the non-gout cohort (median age 62 and 59 years, respectively) as older patients with gout tended to have fewer patients without gout available for matching (see online supplementary table 1 ). The overall proportion of men was similar between cohorts $(76.7 \%$ among gout cohort, $77.1 \%$ among non-gout cohort). Distribution of baseline characteristics was similar between the 623861 patients with gout and without included in the final study population and the 1012471 patients with gout and without in the original cohort who met eligibility criteria but had no linked HES or ONS data available (see online supplementary table 2 ).

\section{Risk of advanced CKD}

During 3.7 years of median follow-up (IQR, 1.7-7.0) there were 3452 incident CKD events among 404035 patientyears in the gout cohort and 10187 incident events among 2498498 patient-years in the cohort without gout (table 1). The unadjusted analysis found that patients with gout experienced 2.00 (95\% CI 1.92 to 2.07) times the risk of advanced CKD relative to those without gout (table 1 and illustrated in figure 3). With adjustment for potential confounders, the risk was attenuated but remained statistically significant (HR, 1.29; 95\% CI 1.23 to $1.35, \mathrm{p}<0.001)$.

For each component of the primary outcome, incidence rates of CKD were consistently higher among patients with gout compared with those without gout although the magnitude of the associations varied (table 1). For the outcome of ESKD, the greatest magnitude of association was observed with patients with gout having 2.13 (95\% CI 1.73 to 2.61) times the risk of incident ESKD compared with patients without gout following adjustment. The pattern was similar for other component parts of the composite outcome (eGFR $<10 \mathrm{~mL} / \mathrm{min} / 1.73 \mathrm{~m}^{2}$ (HR, 1.45 ; 95\% CI 1.30 to 1.61 ) and doubling of serum creatinine (HR, 1.13 ; $95 \%$ CI 1.08 to 1.19 ), although the magnitude of the relationship was less. The association of gout with death associated with CKD did not achieve statistical significance (HR, 1.14; 95\% CI 0.99 to 1.31 ).

\section{Sensitivity analyses}

Sensitivity analyses yielded associations between gout and advanced CKD that were similar to the main analysis; adjusted HR 1.28 (95\% CI 1.22 to 1.35 ) for incident patients with gout compared with matched patients without gouts and 1.23 (95\% CI 1.17 to 1.29) among propensity score-matched patients with gout and without (see online supplementary figure 1 ).

\section{Subgroup analyses}

The association of gout and advanced CKD varied when stratified by the presence or absence of comorbidities (figure 4). While the level of statistical evidence varied, overall the gout cohort had higher risk of advanced CKD than the non-gout cohort across all groups with and without history of CKD (no CKD: HR, 1.46; 95\% CI 1.31 to 1.64; early-stage CKD: HR, 1.07; $95 \%$ CI 0.96 to 1.19 ; moderate-stage CKD: HR, 1.18; 95\% CI 1.06 to 1.31 ). The highest risk was observed among patients with history of heart failure (HR, 1.84; 95\% CI 1.00 to 3.38), although the CI was wide, followed by those without the underlying condition (HR, $1.21 ; 95 \%$ CI 1.14 to 1.28). There was no evidence of an association between gout and advanced CKD among patients with a history of diabetes (HR, 0.96; $95 \%$ CI 0.77 to 1.19 ); however, patients with gout but without diabetes had higher risk of advanced CKD compared with patients without gout (HR, 1.31; 95\% CI 1.24 to 1.40$)$.

\section{DISCUSSION}

This large observational study provides strong evidence of an association between gout and progression to advanced CKD in the real-world setting of routine clinical practice in the UK health system between January 2000 and July 2015. After accounting for differences between the two cohorts, patients with gout had a $29 \%$ higher risk of advanced CKD than patients without gout (HR, 1.29, 95\% CI 1.23 to 1.35$)$. These findings were consistent in sensitivity analyses conducted among patients newly diagnosed with gout (HR, 1.28; 95\% CI 1.22 to 1.35 ) and among a propensity score-matched subpopulation (HR, $1.23 ; 95 \% \mathrm{CI} 1.17$ to 1.29$)$. Of the four components of the primary outcome, the highest risk was observed for ESKD (HR, 2.13; 95\% CI 1.73 to 2.61). The risk was also present for several surrogate measures of CKD progression including a doubling of serum creatinine and progression to a GFR value $<10 \mathrm{~mL} / \mathrm{min} / 1.73 \mathrm{~m}^{2}$. The association remained when stratified by the presence or absence of early-stage CKD and heart failure, although no association was observed in patients with diabetes. 


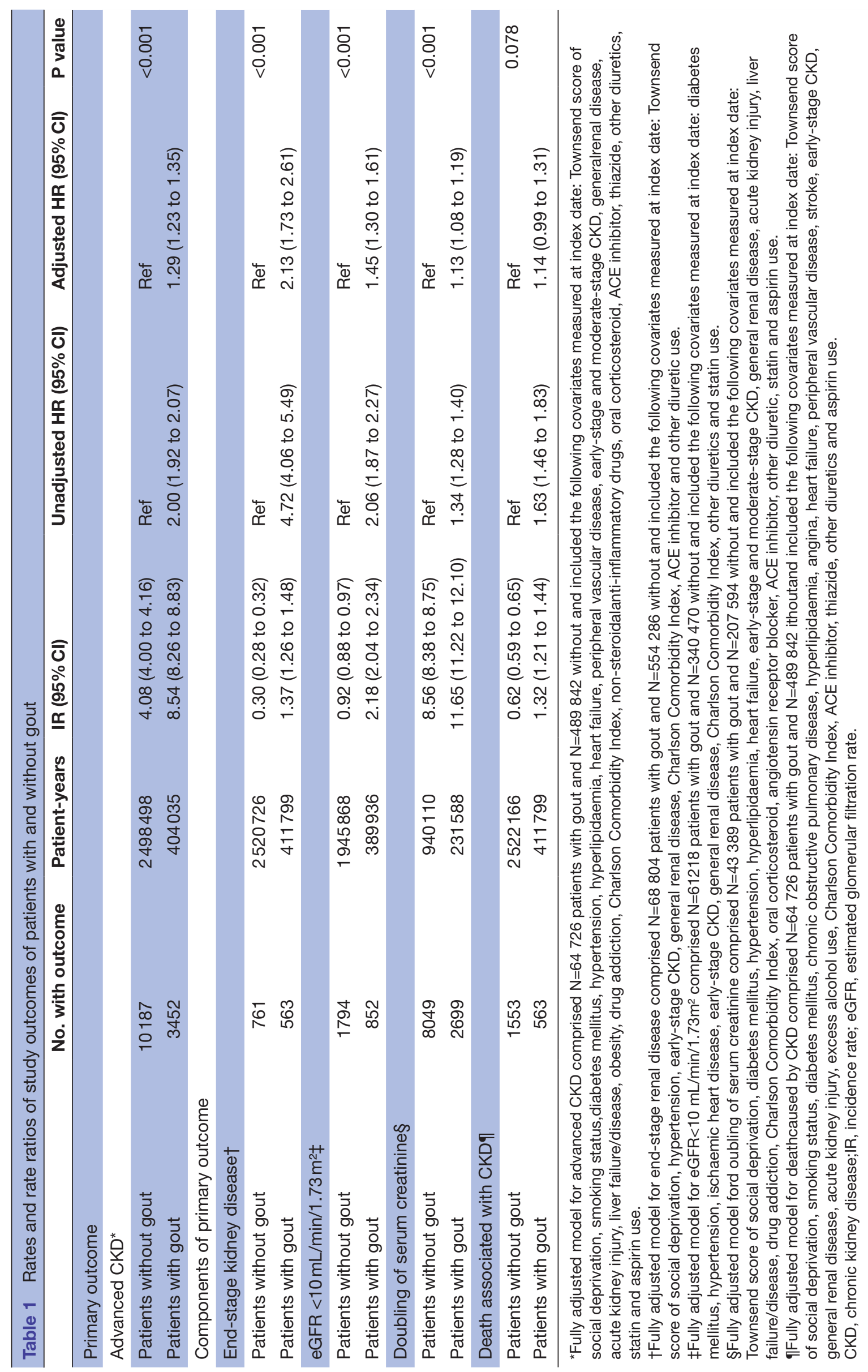




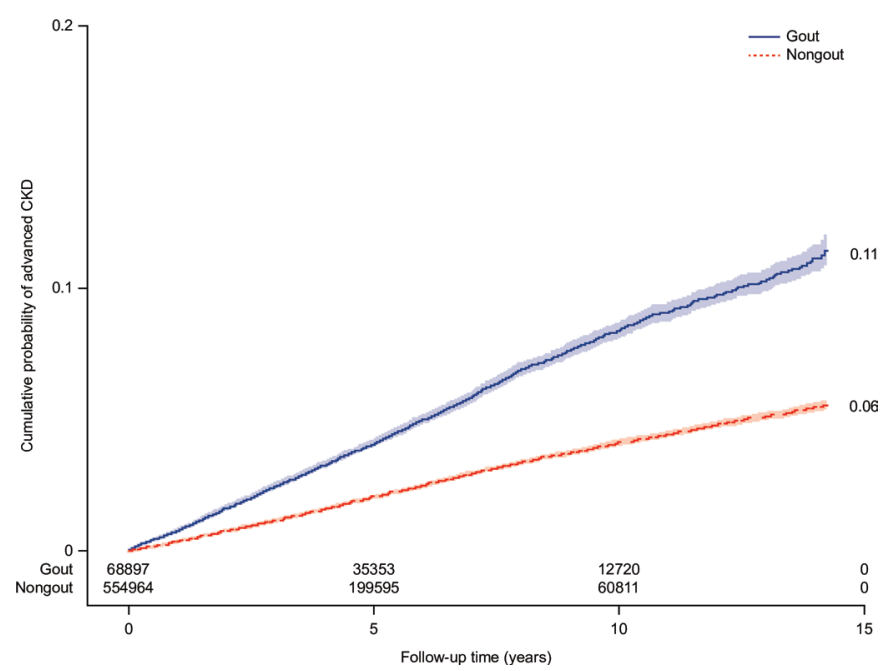

Figure 3 Cumulative probability of advanced chronic kidney disease among patients with and without gout. CKD, chronic kidney disease.

This is perhaps due to the high risk of ESKD caused by nephropathy in patients with diabetes ${ }^{29}$ outweighing the risk caused by gout.

Although it is known that the presence of CKD contributes to the progression of hyperuricaemia and gout, the findings from this national observational cohort study of patients in the UK health system demonstrate that gout is also strongly associated with an increased risk of advanced CKD. After adjusting for known confounders, the association of gout with advanced CKD was not explained by differences in demographic or socioeconomic factors, the presence or severity of prevalent comorbid conditions including pre-existing CKD or the use of known nephrotoxic medications. The magnitude of the goutCKD association was greatest for a diagnosis of ESKD with over a twofold higher risk, but the risk was also present for several surrogate measures of CKD progression, including a doubling of serum creatinine and progression

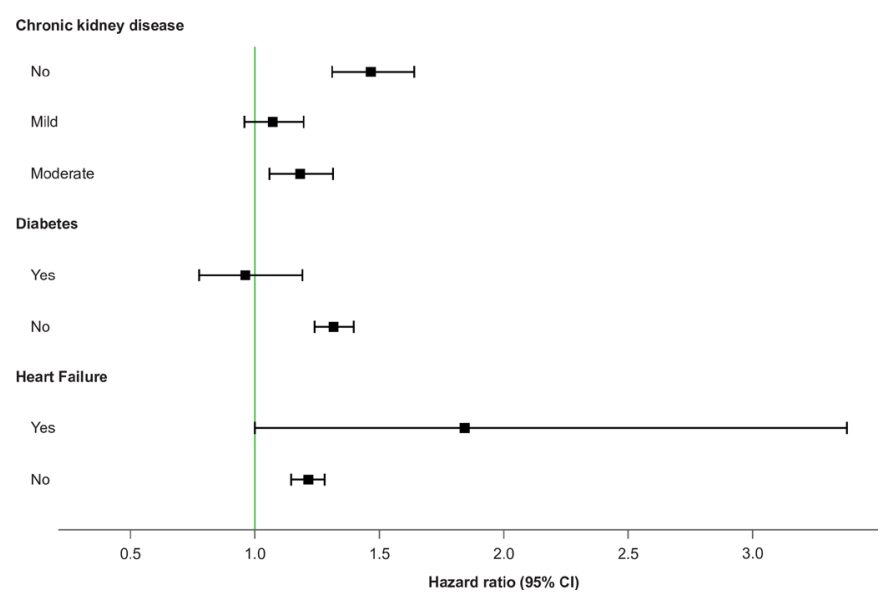

Figure 4 Adjusted HR for the risk of advanced chronic kidney disease for patients with gout versus without among subgroups with history of comorbidities, which were identified a priori as clinically important patient characteristics. to an eGFR value $<10 \mathrm{~mL} / \mathrm{min} / 1.73 \mathrm{~m}^{2}$. These findings were confirmed in propensity-matched analysis and were further validated in sensitivity analysis where we included only patients with incident gout. Taken together, these observations suggest that gout is an independent risk factor for progression to advanced CKD.

This study sheds new light on the pattern and magnitude of association of gout with the risk of CKD in the general population. While previous observational studies have convincingly shown that patients with gout have a high burden of CKD, and risk factors for CKD compared with those without gout, ${ }^{30} 31$ only one study to our knowledge has explored the direct impact of gout on the future risk of advanced CKD. Yu et al using data from the National Taiwanese Health System, found that gout was associated with a $57 \%$ higher hazard of ESKD compared with patients without gout adjusting only for age, sex, diabetes and hypertension; the failure to account for common kidney disease risk factors including nephrotoxic medications was a notable limitation. ${ }^{32}$ In the current analysis, we carefully considered several known CKD risk factors that could confound the association of gout with advanced CKD.$^{33}$ Each multivariable model adjusted for a comprehensive set of explanatory sociodemographic characteristics, kidney-specific factors including nephrotoxic medications and lifestyle factors known to be related to CKD. Yet when we accounted for these factors in adjusted analyses, the pattern of association of gout with advanced CKD remained.

Our analysis suggests a strong relationship between clinical gout, an index of cumulative burden of hyperuricaemia and the risk of progressive CKD. It extends an emerging body of evidence that supports a role for chronic hyperuricaemia in the pathogenesis of CKD. ${ }^{34}$ Experimental studies in animals have demonstrated biological plausibility from uric acid-induced intraglomerular hypertension and tubulointerstitial fibrosis. ${ }^{14} 15$ Observational studies in humans have yielded strong independent associations between hyperuricaemia and hazards of CKD progression including ESKD. ${ }^{35-37}$ Further support of a possible causal relationship stems from quasirandomised studies and clinical trials that suggest a slowing of CKD progression with ULTs ${ }^{38} 39$ and prevention of $\mathrm{ESKD},{ }^{18}$ although these findings require confirmation in large clinical trials. ${ }^{40-42}$

This study is not without limitations. The case definition of gout was based on both physician diagnosis and treatment with ULT rather than on strict clinical and laboratory criteria alone. While we recognise the potential for misclassification, previous studies have shown that this definition has high validity. ${ }^{32}$ Moreover, the completeness and accuracy of CPRD data has been confirmed with Read code diagnoses of gout having a $90 \%$ positive predictive value. ${ }^{434}$ Even with this broad definition, it is possible that some cases of gout, where poorly recorded or underdiagnosed in the population, would not have been captured. We also submit that variation may exist in the recording of prescriptions, diagnoses and laboratory 
test results between practitioners, primary care practices and over time. This was mitigated through the use of HES-linked data to identify secondary care diagnoses and the 2004 introduction of the Quality and Outcomes Framework that incentivised practices to improve electronic recording. ${ }^{22}$ As with all observational studies, we acknowledge the possibility of residual confounding due to unmeasured characteristics, however, the CPRD captured data on multiple confounders including prevalent comorbid conditions, severity of illness indicators, kidney-specific risk factors, lifestyle traits and socioeconomic factors, which allowed us to examine in detail the independence of the gout-CKD relationship. The availability of data on these factors is potentially subject to selection bias, as patients with gout or CKD would be more likely to interact with the healthcare system. A further source of selection bias could be the exclusion of patients, although the majority of exclusions were unrelated to patient characteristics such as the availability of at least 12 months of historical data and data linkage.

The limitations of this study were counterbalanced by several strengths. The large sample size of over 600000 patients and moderate period of follow-up provided a powerful mechanism to examine multiple outcomes including ESKD. The study cohort analysed data from CPRD,${ }^{22}$ ensuring that our results are broadly generalisable to primary care practices in the UK health system population. The availability of detailed data on common kidney disease risk factors including nephrotoxic medications provided us with an additional opportunity to account for the influence of these factors in the goutCKD relationship. Recognising that patients with gout had higher burden of comorbid disease and risk factors, we adjusted for an extensive set of baseline characteristics in the primary analysis, conducted additional propensity score sensitivity analysis and repeated the entire analysis in patients exposed to incident gout. In each of these analyses, the independent association of gout with advanced CKD was confirmed following covariate adjustment.

We conclude that gout is independently associated with the risk of advanced CKD in the UK health system. The risk was greatest for the outcome of ESKD but extended to other surrogate measures of CKD progression. This relationship was not explained by prevailing burden of medical conditions, lifestyle factors, NSAID use or differences in socioeconomic factors measured at baseline. The overall findings add to the accumulating body of evidence that gout is an independent risk factor for kidney disease progression, although further work is needed to establish whether adequate control of gout can reduce the risk of CKD progression.

\section{Author affiliations}

${ }^{1}$ Graduate Entry Medical School \& Health Research Institute, University of Limerick, Limerick, Ireland

${ }^{2}$ OXON Epidemiology, London, UK

${ }^{3}$ AstraZeneca, Luton, UK

${ }^{4}$ AstraZeneca, Gaithersburg, Maryland, USA

${ }^{5}$ YourCareChoice, Ann Arbor, Michigan, USA
Contributors AGS, RM and BB conceived the study protocol. AGS, BB, MEJ, AK, RM and ARM coordinated the execution of the study. VLP, MEJ and LC performed the statistical analyses. AGS, VLP and MEJ drafted the manuscript. All authors contributed to the interpretation of results, further drafts of the manuscript and approved the final manuscript.

Funding statement This study was funded by AstraZeneca. BB, AK, and RM were employed by AstraZeneca the time of the study and were involved in the design of the study, interpretation of the results, the preparation, review, and approval of the manuscript and the decision to publish.

Competing interests AGS received funding from AstraZeneca to design the study protocol and provide scientific expertise. VLP, MEJ, LC and ARM were employed by OXON Epidemiology Ltd at the time of the study, which has received funding from AstraZeneca to conduct the study and develop the manuscript. BB, AK and RM were employed by AstraZeneca at the time of the study, which funded the study.

Patient consent for publication Not required.

Ethics approval The protocol for this research was approved by the Independent Scientific Advisory Committee (ISAC) of the Medicines and Healthcare Products Regulatory Agency (protocol number 15_009RMn). The CPRD has been granted generic ethics approval for observational studies that make use of only anonymised data and linked anonymised NHS healthcare data (Multiple Research Ethics Committee ref. 05/MRE04/87).

Provenance and peer review Not commissioned; externally peer reviewed.

Data availability statement MEJ had full access to all the data in the study and takes responsibility for the integrity of the data and the accuracy of the data analysis. All source data were obtained from the Clinical Practice Research Datalink (CPRD), linked with data from Hospital Episodes Statistics database and Office of National Statistics. These data sources are made available for scientific and medical research after submission of a study protocol to be reviewed and approved by the CPRD Independent Scientific Advisory Committee (ISAC). Owing to ethical restrictions, the data used in this analysis are not publicly available, in line with the data privacy rules set up by CPRD/ISAC.

Open access This is an open access article distributed in accordance with the Creative Commons Attribution Non Commercial (CC BY-NC 4.0) license, which permits others to distribute, remix, adapt, build upon this work non-commercially, and license their derivative works on different terms, provided the original work is properly cited, appropriate credit is given, any changes made indicated, and the use is non-commercial. See: http://creativecommons.org/licenses/by-nc/4.0/.

\section{REFERENCES}

1. Ayodele OE, Alebiosu CO. Burden of chronic kidney disease: an international perspective. Adv Chronic Kidney Dis 2010;17:215-24.

2. Coresh J, Selvin E, Stevens LA, et al. Prevalence of chronic kidney disease in the United States. JAMA 2007;298:2038-47.

3. Go AS, Chertow GM, Fan D, et al. Chronic kidney disease and the risks of death, cardiovascular events, and hospitalization. $N$ Engl J Med 2004;351:1296-305.

4. Chonchol M, Shlipak MG, Katz R, et al. Relationship of uric acid with progression of kidney disease. Am J Dis 2007;50:239-47.

5. Madero $M$, Sarnak MJ, Wang X, et al. Uric acid and long-term outcomes in CKD. Am J Dis 2009;53:796-803.

6. Weiner DE, Tighiouart $\mathrm{H}$, Elsayed EF, et al. Uric acid and incident kidney disease in the community. J Am Soc Nephrol 2008;19:1204-11.

7. Krishnan E. Reduced glomerular function and prevalence of gout: NHANES 2009-10. PLoS One 2012;7:e50046.

8. , et alPrevalence of CKD and uncontrolled gout among US adults: results from NHANES 2007-2012. Am J Kidney Dis 2017;69.

9. Kuo C-F, Grainge MJ, Mallen C, et al. Eligibility for and prescription of urate-lowering treatment in patients with incident gout in England. JAMA 2014;312:2684-6.

10. Becker MA, Fitz-Patrick D, Choi HK, et al. An open-label, 6-month study of allopurinol safety in gout: the LASSO study. Semin Arthritis Rheum 2015;45:174-83.

11. Becker MA, Schumacher HR, Espinoza LR, et al. The uratelowering efficacy and safety of febuxostat in the treatment of the hyperuricemia of gout: the confirms trial. Arthritis Res Ther 2010;12.

12. Becker MA, Schumacher HR, Wortmann RL, et al. Febuxostat compared with allopurinol in patients with hyperuricemia and gout. $N$ Engl J Med 2005;353:2450-61.

13. Schumacher HR, Becker MA, Wortmann RL, et al. Effects of febuxostat versus allopurinol and placebo in reducing serum urate 
in subjects with hyperuricemia and gout: a 28-week, phase III, randomized, double-blind, parallel-group trial. Arthritis Rheum 2008;59:1540-8.

14. Mazzali M, Hughes J, Kim YG, et al. Elevated uric acid increases blood pressure in the rat by a novel crystal-independent mechanism. Hypertension 2001;38:1101-6.

15. Mazzali M, Kanellis J, Han L, et al. Hyperuricemia induces a primary renal arteriolopathy in rats by a blood pressure-independent mechanism. Am J Physiol Renal Physiol 2002;282:F991-F997.

16. Cea Soriano L, Rothenbacher D, Choi HK, et al. Contemporary epidemiology of gout in the UK general population. Arthritis Res Ther 2011;13.

17. Kuo C-F, Grainge MJ, Mallen C, et al. Rising burden of gout in the UK but continuing suboptimal management: a nationwide population study. Ann Rheum Dis 2015;74:661-7.

18. Levy GD, Rashid N, Niu F, et al. Effect of urate-lowering therapies on renal disease progression in patients with hyperuricemia. $J$ Rheumatol 2014;41:955-62.

19. Bardin T, Richette P. Impact of comorbidities on gout and hyperuricaemia: an update on prevalence and treatment options. BMC Med 2017;15:123.

20. Perez-Ruiz F, Calabozo M, Herrero-Beites AM, et al. Improvement of renal function in patients with chronic gout after proper control of hyperuricemia and gouty bouts. Nephron 2000;86:287-91.

21. Richette P, Perez-Ruiz F, Doherty M, et al. Improving cardiovascular and renal outcomes in gout: what should we target? Nat Rev Rheumatol 2014;10:654-61.

22. Herrett E, Gallagher AM, Bhaskaran K, et al. Data resource profile: clinical practice research Datalink (CPRD). Int J Epidemiol 2015;44:827-36.

23. Levey AS, Stevens LA, Schmid $\mathrm{CH}$, et al. A new equation to estimate glomerular filtration rate. Ann Intern Med 2009;150:604-12.

24. Matsushita K, Mahmoodi BK, Woodward M, et al. Comparison of risk prediction using the CKD-EPI equation and the MDRD study equation for estimated glomerular filtration rate. JAMA 2012;307:1941-51.

25. Matsushita K, Selvin E, Bash LD, et al. Risk implications of the new CKD epidemiology collaboration (CKD-EPI) equation compared with the MDRD study equation for estimated GFR: the Atherosclerosis risk in communities (ARIC) study. Am J Kidney Dis 2010;55:648-59.

26. KDIGO clinical practice guideline for anemia in chronic kidney disease. Kidney Int Supp/ 2012;2:331-5.

27. Hole B, Gilga J, Casula A, et al. Uk renal registry 20th annual report: chapter 1 UK renal replacement therapy adult incidence in 2016: national and Centre-specific analyses. Nephron 2018;139:13-46.

28. Khan NF, Perera R, Harper S, et al. Adaptation and validation of the Charlson index for Read/OXMIS coded databases. BMC Fam Pract 2010;11:1.
29. Ritz E, Rychlík I, Locatelli F, et al. End-Stage renal failure in type 2 diabetes: a medical catastrophe of worldwide dimensions. Am J Kidney Dis 1999;34:795-808.

30. Stack AG, Hanley A, Casserly LF, et al. Independent and conjoint associations of gout and hyperuricaemia with total and cardiovascular mortality. QJM 2013;106:647-58.

31. Zhu Y, Pandya BJ, Choi HK. Comorbidities of gout and hyperuricemia in the US general population: NHANES 2007-2008. Am J Med 2012;125:679-87.

32. KH Y, Kuo CF, Luo SF, et al. Risk of end-stage renal disease associated with gout: a nationwide population study. Arthritis Res Ther 2012;14.

33. Kazancioğlu R. Risk factors for chronic kidney disease: an update. Kidney Int Suppl 2013;3:368-71.

34. Johnson RJ, Nakagawa T, Jalal D, et al. Uric acid and chronic kidney disease: which is chasing which? Nephrology Dialysis Transplantation 2013;28:2221-8.

35. Hsu C-yuan, Iribarren C, McCulloch CE, et al. Risk factors for end-stage renal disease: 25-year follow-up. Arch Intern Med 2009;169:342-50.

36. Iseki K, Ikemiya Y, Inoue T, et al. Significance of hyperuricemia as a risk factor for developing ESRD in a screened cohort. Am J Kidney Dis 2004:44:642-50.

37. Tsai C-W, Lin S-Y, Kuo C-C, et al. Serum uric acid and progression of kidney disease: a longitudinal analysis and mini-review. PLoS One 2017;12:e0170393.

38. Goicoechea M, de Vinuesa SG, Verdalles U, et al. Effect of allopurino in chronic kidney disease progression and cardiovascular risk. Clin J Am Soc Nephrol 2010;5:1388-93.

39. Sircar D, Chatterjee S, Waikhom R, et al. Efficacy of febuxostat for slowing the GFR decline in patients with CKD and asymptomatic hyperuricemia: a 6-month, double-blind, randomized, placebocontrolled trial. Am J Kidney Dis 2015;66:945-50.

40. Clinical Trials New Zealand. Drugs as treatment for chronic kidney disease stages 3 and 4, sponsored by Australasian Kidney Trials Network (University of QId) - New Zealand Clinical Trial \#5448; 2018. http://clinicaltrials.health.nz/trial/

41. Clinicaltrials.gov. A multicenter clinical trial of allopurinol to prevent kidney function loss in type 1 diabetes; 2018. https://clinicaltrials. gov/ct2/show/NCT02017171

42. Hosoya T, Kimura K, Itoh S, et al. The effect of febuxostat to prevent a further reduction in renal function of patients with hyperuricemia who have never had gout and are complicated by chronic kidney disease stage 3: study protocol for a multicenter randomized controlled study. Trials 2014;15:26.

43. Herrett E, Thomas SL, Schoonen WM, et al. Validation and validity of diagnoses in the general practice research database: a systematic review. Br J Clin Pharmacol 2010;69:4-14.

44. Meier $\mathrm{CR}$, Jick H, Omeprazole JH. Omeprazole, other antiulcer drugs and newly diagnosed gout. Br J Clin Pharmacol 1997;44:175-8. 